“(C) 2015 IEEE. Personal use of this material is permitted. Permission from IEEE must be obtained for all other uses, in any current or future media, including reprinting/republishing this material for advertising or promotional purposes, creating new collective works, for resale or redistribution to servers or lists, or reuse of any copyrighted component of this work in other works." 


\title{
Prediction of Freezing of Gait Using Analysis of Brain Effective Connectivity
}

\author{
A.M. Ardi Handojoseno ${ }^{1}$, Student Member, IEEE, James M. Shine ${ }^{2}$, Moran Gilat ${ }^{2}$, \\ Tuan N. Nguyen ${ }^{1}$, Member IEEE, Yvonne Tran ${ }^{1,3}$, \\ Simon J.G. Lewis ${ }^{2}$, Hung T. Nguyen ${ }^{1}$, Senior Member, IEEE
}

\begin{abstract}
Freezing of gait (FOG) is a debilitating symptom of Parkinson's disease (PD), in which patients experience sudden difficulties in starting or continuing locomotion. It is described by patients as the sensation that their feet are suddenly glued to the ground. This, disturbs their balance, and hence often leads to falls. In this study, directed transfer function (DTF) and partial directed coherence (PDC) were used to calculate the effective connectivity of neural networks, as the input features for systems that can detect FOG based on a Multilayer Perceptron Neural Network, as well as means for assessing the causal relationships in neurophysiological neural networks during FOG episodes. The sensitivity, specificity and accuracy obtained in subject dependent analysis were $82 \%$, $\mathbf{7 7 \%}$, and $\mathbf{7 8 \%}$, respectively. This is a significant improvement compared to previously used methods for detecting FOG, bringing this detection system one step closer to a final version that can be used by the patients to improve their symptoms.
\end{abstract}

\section{INTRODUCTION}

Freezing of gait (FOG) is a common symptom affecting more than $70 \%$ of advance Parkinson disease (PD) patients [1]. It is characterized by an "episodic" incapability to start walking, suddenly failing to continue to move forward, a reduction of forward progression of the feet so that the patient "shuffles" forwards (the festination), or a complete absence of movement despite the intention to walk (akinesia) [2]. Even a brief FOG episode may lead to falls, affecting a patients' level of activity and reducing quality of life [3]. It is resistant to pharmacological treatment, especially in the advanced stages of the disease [4], making FOG prediction systems highly important.

Early detection of FOG episodes has already been developed by our group by analyzing energy, entropy and correlations of electroencephalography (EEG) signals. This system enabled to detect FOG with a sensitivity of $83 \%$, however the specificity was only 58\% and accuracy $70 \%$ [5]. Compared to motion sensors, such as accelerometers

${ }^{1}$ A.M. Ardi Handojoseno, Tuan N. Nguyen and Hung T. Nguyen are with Faculty of Engineering and Information Technology, University of Technology, Sydney, Broadway, NSW 2007, Australia. (AluysiusMariaArdi.Handojoseno@student.uts.edu.au,TuanNghia.Nguyen@uts.edu.au, Hung.Nguyen@uts.edu.au)

${ }^{2}$ James M. Shine, Moran Gilat and Simon J.G. Lewis are with Parkinson's Disease Research Clinic, Brain and Mind Research Institute, University of Sydney, Level 4, Building F, 94 Mallet Street, Camperdown, NSW, 2050, Australia. (mac.shine@sydney.edu.au, moran.gilat@sydney.edu.au, simonl@med.usyd.edu.au)

${ }^{3}$ Yvonne Tran is with the Key University Research Centre for Health Technologies, University of Technology, Sydney and the Rehabilitation Studies Unit, University of Sydney, Australia. (Yvonne.Tran@uts.edu.au) or EMG [6], EEG has an advantage in its ability to track the physiological process of freezing from the earliest stage throughout the analysis of brain dynamics, which also provides insights into possible pathophysiological mechanisms underlying neurological development and disease.

It is known that neural connectivity plays a crucial role in determining the functional properties of neurons and neuronal system. Therefore, the concept of brain connectivity is central for our understanding of organized behaviour of cortical regions beyond the simple mapping of their activity. It has been successfully used to study neurological conditions and disorders, such as Autism [7], Alzheimer's disease [8], and Schizophrenia [9]. In this paper, directed transfer function (DTF) and partial directed coherence (PDC) were used to investigate the connectivity pattern of PD patients' brain area's of interest during freezing episodes. DTF and PDC were used due to their reliability and robustness in neuronal directionality assessment, beyond correlational analyses [10]. The measures of DTF and PDC were taken as the input for the Multilayer Perceptron Neural Network (MLP-NN) to detect the transition of brain signals before freezing episodes.

\section{METHODS}

\section{A. Data Collection and Preprocessing}

The EEG data used in our study were collected from 10 patients (age $75.1 \pm 6.3$ ) with idiopathic Parkinson's disease and significant FOG as measured during a structured series of video-recorded timed up-and-go tasks (TUG). The patients were recruited from the Parkinson's Disease Research Clinic at the Brain and Mind Research Institute, University of Sydney. EEG data were acquired using a 4 channel wireless EEG system with gold cup electrodes. Based on their role in control movement, the electrodes were placed at the following bipolar EEG channels: O1-T4 (visual), P4-T3 (sensorimotor affordance), $\mathrm{Cz}-\mathrm{FCz}$ (motor execution) and $\mathrm{Fz}-\mathrm{FCz}$ (motor planning). The recordings were bandpass filtered between 0.15 and $100 \mathrm{~Hz}$ and were segmented to 1 -s durations and digitized at 500 samples per second.

Two physicians specialized in movement disorders inspected and labeled the start and duration of the freezing episodes. Based on this analysis, two other groups were determined: normal walking data and transition data (5 seconds before freezing), as has been reported elsewhere [5]. After removing the EEG data segment that were affected by artifact using visual inspection, 843 selected samples data 
were filtered from the low and high frequency noise and 50 $\mathrm{Hz}$ line frequency using band-pass $(0.5-60 \mathrm{~Hz})$ and bandstop $(50 \mathrm{~Hz})$ Butterworth IIR with zero phase shift. The EEG data were normalized with a z-transformation to eliminated differences in source strength due to inter-individual and inter-electrode variance in absolute measurements.

\section{B. Multivariate Autoregressive Process}

DTF and PDC estimations are based on the application of the Granger causality into multivariate autoregressive (MVAR) models of time series. They are defined as a frequency domain representation of the existing multivariate relationships between simultaneously analyzed time series, which provide a linear measures of causality, indicating the direction and strength of the interactions between multiple coupled variables [11]. When considering a set of stationary EEG signals time series $X(t)=X_{1}, X_{2}, \ldots, X_{N}(N$ EEG signals simultaneously observed), then the MVAR model is defined as:

$$
\left[\begin{array}{c}
x_{1}(t) \\
\cdot \\
\cdot \\
\cdot \\
x_{N}(t)
\end{array}\right]=\sum_{r=1}^{p} A_{r}\left[\begin{array}{c}
x_{1}(t-r) \\
\cdot \\
\cdot \\
\cdot \\
x_{N}(t-r)
\end{array}\right]+\left[\begin{array}{c}
w_{1}(t) \\
\cdot \\
\cdot \\
\cdot \\
w_{N}(t)
\end{array}\right]
$$

where $A_{r}$ are the model coefficients, $p$ is the model order, and $w_{i}(t)$ represents a random Gaussian white noise. The model order $p$ was estimated by means of Schawarz's Bayesian Criterion (SBC) due to its robustness [12]. The Nuttall Strand method (multivariate Burg) which has been reported as superior to other methods was used to estimate the MVAR parameters [13].

The time domain representation was then translated to frequency domain by computing the cross-spectral power density matrix:

$$
S(f)=H(f) \sum H^{H}(f)
$$

where the superscript $H$ indicates the Hermitian transpose and $H(f)$ is a transfer function matrix

$$
H(f)=A^{-1}(f)=[I-A(f)]^{-1}
$$

where

$$
A(f)=I-\sum_{r=1}^{p} A_{r} e^{-2 i \pi f r}
$$

is the Fourier transform of the model coefficient matrix.

\section{Directed Transfer Function}

The directed transfer function (DTF) estimates the causal influence of the cortical waveform in the channel $j$ on channel $i$ at a certain frequency $f$, and normalized by dividing the inflow from channel $j$ to channel $i$ by all the inflows to channel $i$. It is defined as [14]

$$
D T F_{j \rightarrow i}^{2}(f)=\frac{\left|H_{i j}(f)\right|^{2}}{\sqrt{\sum_{k=1}^{N}\left|H_{i k}(f)\right|^{2}}} .
$$

This normalization resulted in the interval $[0,1]$ values, with 1 indicating that all of the signal in channel $i$ is
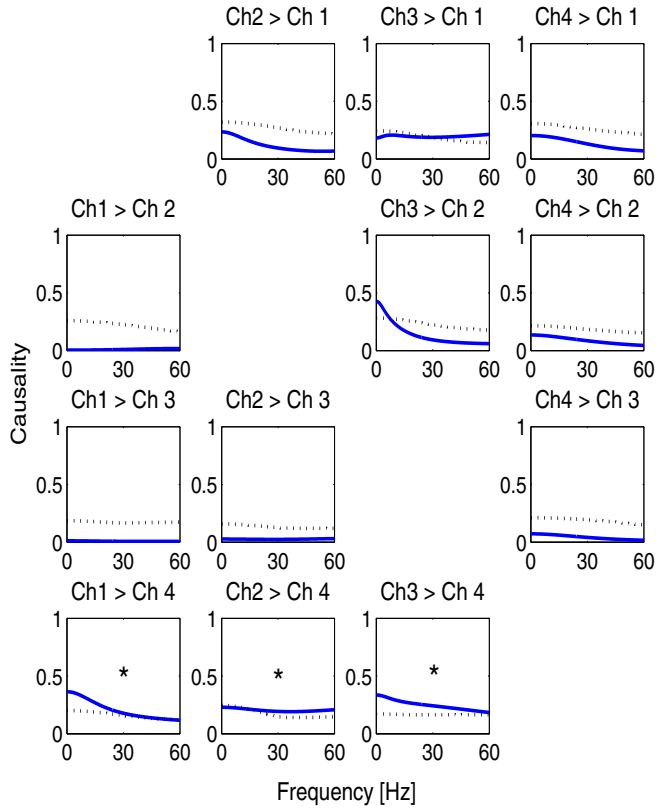

Fig. 1. Sample of estimated sGPDC values (solid lines) and their corresponding surrogates as a threshold of significance estimation (dotted lines), between O1 (channel 1), P4 (channel 2), Cz (channel 3) and P4 (channel 4) from patient 9 during transition preceding the FOG. Only values between $0 \mathrm{~Hz}$ and $60 \mathrm{~Hz}$ were shown due to physiological significance. An asterisk indicates channel pairs with significant sGPDC causality.

caused by the signal from channel $\mathrm{j}$. The denominator of this ratio depends on the frequency, making it difficult when comparing the outflows at different frequencies. Besides, it does not distinguish between direct and cascade flows.

For estimation of direct connections, different normalization has been introduced as the full frequency Directed Transfer Function (ffDTF) [14]

$$
f f D T F_{j \rightarrow i}^{2}(f)=\frac{\left|H_{i j}(f)\right|^{2}}{\sqrt{\sum_{f} \sum_{k=1}^{N}\left|H_{i k}(f)\right|^{2}}} .
$$

When multiplied by the partial coherence, this modification of DTF results in the direct DTF (dDTF) [14]:

$$
d D T F_{j \rightarrow i}^{2}(f)=f f D T F i j(f) P C o h_{i j}(f)
$$

where

$$
\operatorname{PCoh}_{i j}^{2}(f)=\frac{M_{i j}^{2}(f)}{M_{i i}(f) M_{j j}(f)}
$$

and $M_{i j}(f)$ is the minor obtained by removing $i$ th row and $j$ th column from the spectral matrix $S$.

\section{Partial Directed Coherence}

The partial directed coherence (PDC) was introduced to improve and add more information to DTF by distinguishing direct from indirect flow between channels. It does not involve the inversion of matrix A, making it computationally more efficient and precise compared to DTF. With $\bar{A}_{i, j}$ being the $i, j$ th element of $\bar{A}(f)$, the PDC from channel $j$ to $i$ could be calculated as [15] 


$$
P D C_{j \rightarrow i}(f)=\frac{\bar{A}_{i j}(f)}{\sqrt{\sum_{k=1}^{N} \bar{A}_{k i}(f) \bar{A}_{k j}^{*}(f)}} .
$$

As in the DTF, the PDC has values between 0 and 1, with higher values indicating a higher strength of signals transmission from channel $\mathrm{j}$ to $\mathrm{i}$ at this frequency.

PDC emphasizes the sinks rather than the sources as it is normalized to show a ratio between the outflow from channel $\mathrm{j}$ to channel $\mathrm{i}$ to all the outflows from the source channel $\mathrm{j}$. Normalization factor was modified in the squared generalized PDC to make PDC scale-invariant, has an absolute strength of the coupling, emphasizes the sources, and has a greater sensitivity[15] [10]:

$$
s G P D C_{j \rightarrow i}(f)=\frac{\left(\frac{1}{\sigma_{i}}\left|\bar{A}_{i j}(f)\right|\right)^{2}}{\sum_{k=1}^{N} \frac{1}{\sigma_{k}^{2}} \bar{A}_{k j}(f) \bar{A}_{k j}^{*}(f)} .
$$

Signals from four EEG electrodes were included in the MVAR model. The short-time DTF, and PDC method was used to measure the connectivity of the data due to its ability to tract fast changes in the brain signals [12]. A data epoch of one second was divided into short overlapping time windows, with a window length of $256 \mathrm{~ms}$ and a quarter overlap window. The DTF, dDTF, PDC, and sGPDC were computed in each window over the $1-60 \mathrm{~Hz}$ frequency range, and were analyzed in five clinical EEG frequency bands: delta, theta, alpha, beta and gamma. A surrogate data method based on Theiler algorithm with 20 realizations was used to select only a directed causal influence, which has less than $1 \%$ probability occurring by chance [14]. Surrogates, which indicated the 'leak flows' between channels, were produced by randomizing the signal so that they maintained the spectral properties of the original data sample but destroyed nonlinear-phase relations. Fig.1 illustrates sGPDC measures of the EEG data of subject 9 during the transition to freezing.

For connectivity analysis, individual trial DTF, dDTF, PDC and sGPDC spectrograms were averaged within each subject and then averaged across all subjects within each group, for all directions of connectivity between pairs of electrode regions. These measures represent the strength and the number of causal interactions originating at each electrode, which provides a measure of the source activity arising from each node in a network.

\section{E. Statistical Classification}

The mean, the maximum and the minimum values of DTF, dDTF, PDC and sGPDC for each pair of electrodes in each EEG frequency band were taken from two conditions, normal walking and transition to freezing, as features for the prediction of FOG. The non parametric Wilcoxon Sum Rank Test was used to select the most significant feature to feed the classifier for each experiment. A $p$-value $<0.05$ and $r$-value $>2.5$ were chosen for further process.

For classification, MLP-NN was used due to its good results in classification of EEG signals [16]. A three layer Back Propagation Neural Networks with 4 to 5 hidden nodes was built. Bayesian regularization [17] was used to prevent over-fitting and to improve generalization, with $80 \%$ of the

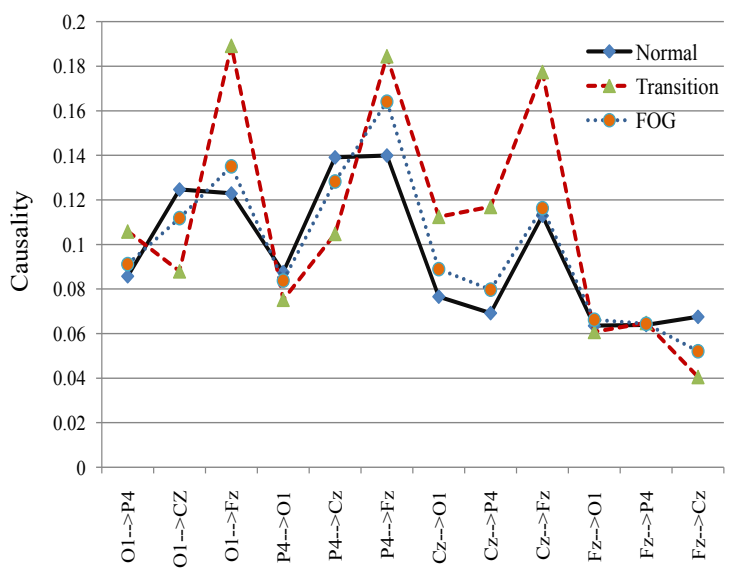

Fig. 2. The mean value of sGPDC from 10 patients which indicates the connection between four locations of EEG electrodes

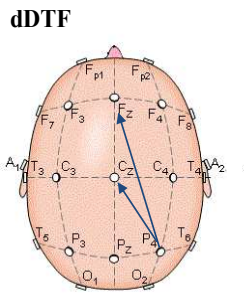

Normal Walking SGPDC

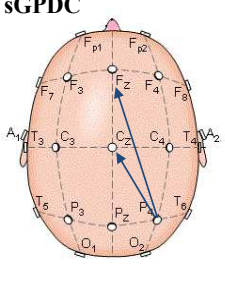

Normal Walking

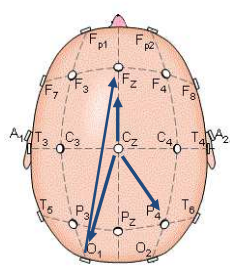

Transition

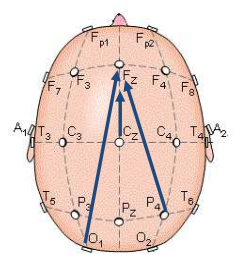

Transition

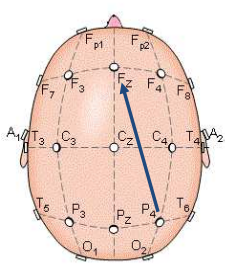

Freezing of Gait

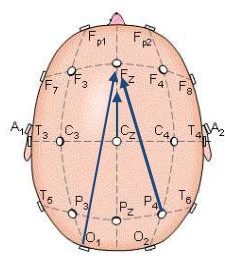

Freezing of Gait
Fig. 3. The schematic interaction between four locations of EEG electrodes during normal walking and FOG estimated using dDTF and sGPDC at theta band frequency. The arrow line width shows the connectivity strength.

data trained by Levenberg Marquardt algorithm and $20 \%$ of the data being used for testing. Mean squared prediction error was used to measure the prediction of sensitivity, specificity and accuracy. The average and standard deviation of fifty training and testing were recorded for further analysis.

\section{RESULT AND DISCUSSION}

The first row of Fig. 1 indicates a lot of noise on the electrode which picks signals from the occipital one (channel 1), making the "leak inflows" from this electrode relatively high. When only directed causal influence which were higher than the threshold were taken, we noticed that FOG episodes were characterised by abnormal EEG hyperconnectivity involving the frontal region. It received stronger outflows connection from other regions (Fig. 2 and Fig. 3), especially in the lower frequency bands (the theta and alpha band). This suggests that the frontal region, which supports spatial attention, motor intention, cognitive and decision making processes is prominently involved in generating the hypersynchronization 
TABLE I

CLASSIFICATION RESULTS OF PROPOSED FEATURES USING MLP-NN IN DETECTING TRANSITION 5 SECOND BEFORE FREEZING

\begin{tabular}{ccccccc}
\hline \hline Features & \multicolumn{3}{c}{ training } & \multicolumn{3}{c}{ testing } \\
& $\begin{array}{c}\text { Sens } \\
\%\end{array}$ & $\begin{array}{c}\text { Spec } \\
\%\end{array}$ & $\begin{array}{c}\text { Acc } \\
\%\end{array}$ & $\begin{array}{c}\text { Sens } \\
\%\end{array}$ & $\begin{array}{c}\text { Spec } \\
\%\end{array}$ & $\begin{array}{c}\text { Acc } \\
\%\end{array}$ \\
\hline DTF & 70.62 & 75.49 & 73.09 & 65.80 & 69.52 & 67.40 \\
dDTF & 83.18 & 85.42 & 84.31 & 68.55 & 73.17 & 70.86 \\
PDC & 82.73 & 81.51 & 80.27 & 71.11 & 69.07 & 70.02 \\
SGPDC & 79.96 & 81.62 & 80.80 & 72.76 & 70.28 & 71.67 \\
\hline \multicolumn{5}{l}{ Sens: Sensitivity; Spec: Specificity; Acc: Accuracy }
\end{tabular}

TABLE II

CLASSIFICATION RESULTS OF TESTING DATA USING SGPDC AS A FEATURE FOR INDIVIDUAL SUBJECT IN DETECTING TRANSITION 5 SECOND BEFORE FREEZING

\begin{tabular}{cccccccc}
\hline \hline Subj & $\begin{array}{c}\text { Sens } \\
\%\end{array}$ & $\begin{array}{c}\text { Spec } \\
\%\end{array}$ & $\begin{array}{c}\text { Acc } \\
\%\end{array}$ & Subj & $\begin{array}{c}\text { Sens } \\
\%\end{array}$ & $\begin{array}{c}\text { Spec } \\
\%\end{array}$ & $\begin{array}{c}\text { Acc } \\
\%\end{array}$ \\
\hline 1 & 85.93 & 70.24 & 77.50 & 6 & 85.28 & 74.10 & 75.33 \\
2 & 93.24 & 88.41 & 91.00 & 7 & 96.53 & 95.27 & 94.80 \\
3 & 74.37 & 70.64 & 70.00 & 8 & 83.54 & 87.98 & 86.17 \\
4 & 86.80 & 73.71 & 78.57 & 9 & 60.40 & 63.67 & 59.33 \\
5 & 74.50 & 73.73 & 70.67 & 10 & 81.77 & 75.40 & 77.00 \\
\hline \multicolumn{7}{c}{ Average: Sens: $82.24 \%$, Spec: $77.32 \%$, Acc: $78.04 \%$} \\
\hline
\end{tabular}

Subj: Subject; Sens: Sensitivity; Spec: Specificity; Acc: Accuracy

underlaying FOG. This finding supports the hypotheses that attribute FOG to frontal executive dysfunction and visuospatial impairment in PD patients with FOG [18].

Both $\mathrm{dDTF}$ and SGPDC revealed significant connectivity of $\mathrm{P} 4>\mathrm{Fz}$ and $\mathrm{P} 4>\mathrm{Cz}$ in normal walking. They were also in agreement when indicating $\mathrm{O} 1>\mathrm{Fz}$ and $\mathrm{Cz}>\mathrm{Fz}$ connectivity during transition and a stronger $\mathrm{P} 4>\mathrm{Fz}$ connectivity during freezing episodes. The dDTF analysis shows that the significant outflows from $\mathrm{Cz}$ to the other 3 electrode locations may signify the overload of cognitive resources as a consequence of a loss of automaticity which shifted neural activation from the subcortical (basal ganglia) to more cortical areas [19].

When used as features for the early detection of FOG, both $\mathrm{dDTF}$ and SGPDC show significant increases in performance of the prediction system compared to their original form, DTF and PDC. In addition, sGPDC appears to be the strongest indicator of the transition to freezing with an average sensitivity, specificity, and accuracy of $73 \%, 70 \%$, and $72 \%$, respectively (see Table 1 ). In the subject-dependent analysis where training and testing data were taken from the related subject only, the performance of classification increased to $82 \%, 77 \%$, and $78 \%$ of an average sensitivity, specificity, and accuracy, respectively, with the best results of all performance measures at around $90 \%$ obtained by 2 patients (see Table 2). However, the system achieved the performance of all measures at around $60 \%$ in 1 patient. This indicates the variability in brain connectivity among subjects.

\section{CONCLUSIONS}

This study revealed that the information outflows from the central area during the transition to FOG and the information inflows to the frontal area during freezing episodes were enhanced. This estimation, based on DTF and PDC, also provides useful features for the prediction of FOG, especially in the customized system, with an accuracy of $94.80 \%$ obtained in one patient. The inclusion of more data from more patients, the additional feature selection procedure and the optimization of the algorithm on the brain connectivity estimation and classification will be our future work, to obtain a better performance of this potential approach.

\section{REFERENCES}

[1] I.C. Lopez, P.J.G. Ruiz, S.V.F. del Pozo, and V.S. Bernardos, "Motor complications in Parkinson's disease: Ten year follow-up study," Mov. Disord., vol. 25, no. 16, pp. 2735-2739, 2010.

[2] J.D. Schaafsma, Y. Balash, T. Gurevich, A.L. Bartels, J.M. Hausdorff, and N. Giladi, "Characterization of freezing of gait subtypes and the response of each to levodopa in Parkinson's disease," European J. Neurol., vol. 10, no. 4, pp. 391-398, 2003.

[3] D. Tan, M. Danoudis, J. McGinley, and M.E. Morris, "Relationships between motor aspects of gait impairments and activity limitations in people with Parkinson's disease: a systematic review," Parkinsonism \& Related Disord., vol. 18, no. 2, pp. 117-124, 2012.

[4] N. Giladi, "Medical treatment of freezing of gait," Mov. Disord., vol 23, no. S2, pp. S482-S488, 2008.

[5] A.M. Handojoseno, J.M. Shine, T.N. Nguyen, Y. Tran, S.J.G. Lewis, and H.T. Nguyen, "Using EEG spatial correlation, cross frequency energy, and wavelet coefficients for the prediction of Freezing of Gait in Parkinson's Disease patients," in Proc. 35th Annu. Int. Conf. IEEE Eng. Med. Biol. Soc., 2013, pp. 4263-4266.

[6] M. Bachlin, M. Plotnik, D. Roggen, D. Maidan, J.M. Hausdorff, N. Giladi, and G. Troster, "Wearable Assistant for Parkinsons Disease Patients with the Freezing of Gait Symptom," IEEE Trans. Inform. Tech. Biomed., vol. 14, no. 2, pp. 436-446, 2010.

[7] M.K. Belmonte, G. Allen, A.B. Mitchener, L.M. Boulanger, R.A. Carper, and S.J. Webb, "Autism and abnormal development of brain connectivity," J. Neurosci., vol. 24, no. 42, pp. 9228-9231, 2004.

[8] K. Supekar, V. Menon, D. Rubin, M. Musen, and M.D. Greicius, "Network analysis of intrinsic functional brain connectivity in Alzheimer's disease," PLoS Computational Biology, vol. 4, no. 6, pp.e1000100, 2008

[9] P. Skudlarski, K. Jagannathan, K. Anderson, M.C. Stevens, V.D. Calhoun, B.A. Skudlarska, and G. Pearlson, "Brain connectivity is not only lower but different in schizophrenia: a combined anatomical and functional approach," Biol. Psych., vol. 68, no. 1, pp. 61-69, 2010.

[10] E. Florin, J. Gross, J. Pfeifer, G.R. Fink, and L. Timmermann, "Reliability of multivariate causality measures for neural data," $J$. Neurosci. Methods, vol. 198, no. 2, pp. 344-358, 2011.

[11] L.A. Baccala and K. Sameshima, "Partial directed coherence: a new concept in neural structure determination," Biol. Cyber., vol. 84, no. 6, pp. 463-474, 2001.

[12] C. Porcaro, F. Zappasodi, P.M. Rossini, and F. Tecchio, "Choice of multivariate autoregressive model order affecting real network functional connectivity estimate," Clinic. Neurophysiol., vol. 120, no. 2, pp. 436-448, 2009.

[13] A. Schlogl, "A comparison of multivariate autoregressive estimators," Signal processing, vol. 86, no. 9, pp. 2426-2429, 2006.

[14] R. Kus, M. Kaminski, and K.J. Blinowska, ”Determination of EEG activity propagation: pair-wise versus multichannel estimate," Trans. Biomed. Eng., vol. 51, no. 9, pp. 1501-1510, 2004.

[15] B. Schelter, J. Timmer, and M. Eichler, "Assessing the strength of directed influences among neural signals using renormalized partial directed coherence," J. Neurosci. Methods, vol. 179, no. 1, pp. 121130, 2009.

[16] F. Lotte, M. Congedo, A. Lecuyer, F. Lamarche, and B. Arnaldi. "A review of classification algorithms for EEG-based brain computer interfaces." J. Neural Eng., vol. 4, pp. 1-24, 2007.

[17] F.D. Foresee and M.T. Hagan, "Gauss-Newton approximation to Bayesian learning," in Proc. Int. Conf. Neural Netw., 1997, pp. 19301935.

[18] E. Heremans, A. Nieuwboer, and S. Vercruysse, "Freezing of gait in Parkinsons disease: where are we now?" Current Neurol. Neurosci. Reports, vol. 13, no. 6, pp. 1-9, 2013.

[19] J.M. Shine, S.L. Naismith, and S.J.G. Lewis, "The pathophysiological mechanisms underlying freezing of gait in Parkinsons disease," $J$. Clinic. Neurosci., vol. 18, no. 9, pp. 1154-1157, 2011. 